\title{
EFECTO DE LA LABRANZA MÍNIMA Y LA CONVENCIONAL EN FRIJOL (Phaseolus vulgaris L.) EN LA REGIÓN HUETAR NORTE DE COSTA RICA ${ }^{1}$
}

\author{
Luis Antonio Rojas ${ }^{2}$, Gerardo Chavez ${ }^{2}$
}

\begin{abstract}
RESUMEN
Efecto de la labranza mínima y la convencional en frijol (Phaseolus vulgaris L.) en la Región Huetar Norte de Costa Rica. Se realizó un trabajo en la Región Huetar Norte de Costa Rica en donde se establecieron dos lotes: uno de labranza mínima ( 1,85 ha) y otro de labranza convencional (2 ha). La siembra en ambos sistemas de labranza se realizó con una sembradora abonadora hidraúlica. La variedad sembrada fue la Brunca. La población de plantas de frijol evaluada durante la etapa vegetativa fue de 9,37 plantas/m lineal en labranza mínima, y de 9,55 plantas/m lineal en labranza convencional. La densidad de malezas fue mayor en labranza convencional y las malezas de mayor dominancia fueron $R i$ chardia scabra, Rottboellia cochinchinensis y Spermacoce sp. El peso seco de malezas fue de 37,6 g de malezas/ cuadrícula de $0,25 \mathrm{~m}^{2}$ en labranza mínima, mientras que en el sistema de labranza convencional fue de $46,4 \mathrm{~g}$ de malezas/ cuadrícula. La incidencia y severidad de mustia hilachosa (Tanatheporus cucumeris) en el sistema de labranza mínima fue de un $42,8 \%$ y de 0,83 , respectivamente, mientras que en labranza convencional fue de un $52,2 \%$ y 1,22 , respectivamente. En el sistema de labranza mínima el rendimiento fue de $930 \mathrm{~kg} / \mathrm{ha}$, y en labranza convencional de $676 \mathrm{~kg} / \mathrm{ha}$.
\end{abstract}

\begin{abstract}
Effect of minimun and conventional tillage in bean (Phaseolus vulgaris L.) in the Northern Huetar Region in Costa Rica. The work was conducted in the northern area of Costa Rica where two plots were sown: one of minimum tillage (1.85 ha) and another of conventional tillage (2 ha). Both tillage systems were planted with a hydraulic sower fertilizer. The sown variety was the Brunca. The population of bean plants evaluated during the vegetative stage was of 9.37 plants/lineal meter in minimum tillage, and of 9.55 plants/lineal meter in conventional tillage. The weed density was larger under conventional tillage and the most prevalent weeds were Richardia scabra, Rottboellia cochinchinensis y Spermacoce sp. The dry weight of weeds was of $37.6 \mathrm{~g}$ of weed/frame of $0.25 \mathrm{~m}^{2}$ in minimum tillage, while in the conventional tillage it was of $46.4 \mathrm{~g}$ of weed/frame. The incidence and severity of web-blight (Tanatheporus cucumeris) in the system of minimum tillage was of $42.8 \%$ and of 0.83 , respectively, while in conventional tillage it was of $52.2 \%$ and 1.22 , respectively. In the system of minimum tillage the yield was of $930 \mathrm{~kg} / \mathrm{ha}$, and in conventional of 676 $\mathrm{kg} / \mathrm{ha}$.
\end{abstract}

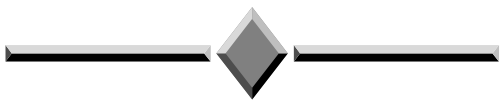

\section{INTRODUCCIÓN}

El cultivo de frijol es una de las actividades agrícolas más importantes en la zona norte de Costa Rica, no obstante, por motivo de la política de apertura comercial de los últimos gobiernos, ha habido una fuerte restricción en la producción de éste y de otros granos (Ledesma 2001).
La Región Huetar Norte en los últimos 10 años suministra más del $50 \%$ de la producción nacional y específicamente en la cosecha 2000/2001 aportó el 61,5\% de la producción (Ledesma 2002).

La producción de cultivos se basa en el manejo del suelo, por lo que es necesario evitar la pérdida de este recurso por efecto de la escorrentía del agua o por el

1 Recibido para publicación el 6 de agosto del 2002.

2 Escuela de Agronomía, Instituto Tecnológico de Costa Rica, Sede Regional San Carlos, Costa Rica. Tel (506)475-50-33 ext 225 ó 216 Fax (506)475-53-95. E-mail: 1rojas@costarricense.cr 
viento (Derpsch 2000). En este sentido, para contrarrestar los efectos del mal uso del suelo que se da en esta región, una gran alternativa es el no laboreo del terreno para la siembra, siembra directa, labranza de conservación o labranza mínima (Gassen y Gassen 1996, Unger et al. 1995, Phillips y Young).

En el sistema de no labranza o labranza mínima se perturba muy poco el suelo y prácticamente la mayor parte del rastrojo de la cosecha anterior queda en la superficie y con ello se evita el proceso de degradación de las propiedades físicas, químicas y biológicas del suelo, con el consecuente peligro de alterar el equilibrio ambiental (Altieri 1985).

Por otra parte, el sistema de labranza en una de las prácticas que más afecta el manejo de malezas ya que además de determinar las especies presentes, afecta la efectividad de los herbicidas y las opciones de manejo (Pitty, 1997). Además, se ha observado un comportamiento irregular entre los sistemas de labranza y la incidencia de plagas y enfermedades en el cultivo de frijol (Vega et al. 1993).

Dentro de un sistema productivo, la labranza mínima ofrece beneficios en el corto, mediano y largo plazo, no obstante, es poca la investigación desarrollada en nuestro país sobre esta tecnología. En vista de lo anterior se realizó este trabajo que es parte de un proyecto de investigación que pretende implementar el uso de la labranza mínima como alternativa de producción sostenible en granos básicos en la Región Huetar Norte.

El objetivo fue evaluar el efecto de la labranza mínima y de la labranza convencional sobre el comportamiento fitosanitario y el rendimiento del cultivo de frijol (Phaseolus vulgaris L.) en la Zona Norte de Costa Rica.

\section{MATERIALES Y MÉTODOS}

El trabajo se realizó en una finca de la localidad de Santa Cecilia de Los Chiles, Alajuela. Según Holdridge (1987), esta zona se clasifica como bosque húmedo tropical.

La siembra del frijol se realizó el 28 de diciembre de 1999. Se debe mencionar que este ciclo de siembra se caracterizó por ser muy lluvioso lo cual afectó el momento de siembra, el desarrollo del cultivo y el rendimiento.

El área experimental constó 1,85 ha sembradas en labranza mínima y 2 ha en labranza convencional.
La variedad de frijol utilizada fue la brunca sembrada a una distancia de $53 \mathrm{~cm}$ entre hileras y de $8 \mathrm{~cm}$ entre plantas. La siembra en ambos sistemas de labranza se realizó con una sembradora de labranza mínima Tatú modelo SDA2E de cuatro líneas de descarga de semilla y fertilizante, con un ancho de labor de 2,4 m y una capacidad de depósito de semilla de $430 \mathrm{~kg}$ y de $680 \mathrm{~kg}$ de fertilizante.

Previo a la siembra del cultivo, la maleza y el rastrojo del cultivo anterior se eliminó con el herbicida glifosato a razón de 3 1/ha.

La máquina de siembra se calibró para sembrar a una distancia de $8 \mathrm{~cm}$ entre plantas lo que dio un promedio de 12,5 semillas/ $\mathrm{m}$ lineal y una densidad de siembra, con base a la distancia de $53 \mathrm{~cm}$ entre hileras, de 235.849 plantas/ha.

En el área de labranza convencional la preparación del terreno se hizo con una pasada de rastra rompedora y luego dos pasadas de rastra afinadora.

Las variables evaluadas fueron: población de plantas de frijol durante la etapa vegetativa evaluada a los 21 días después de la siembra en la etapa denominada V4, población de malezas evaluada previo a la primera aplicación de herbicida posemergente mediante muestreos con cuadrícula de $0,25 \mathrm{~m}^{2}$, peso seco de malezas evaluado al final de la cosecha, incidencia (\% de plantas que presentaron síntomas) y severidad (según la escala del CIAT 1985), evaluada en la etapa de llenado de la vaina (R8), incidencia del barrenador de la vaina (Maruca testulalis) evaluada en R8, número de vainas por planta y número de granos por vaina evaluados en la etapa de maduración de la vaina (R9), y el rendimiento (kg/ha).

Los datos obtenidos en las variables peso seco de malezas, número de vainas por planta, número de granos por vaina, e incidencia y severidad de mustia fueron analizados estadísticamente mediante una prueba de $t$ student $(\mathrm{P} \leq 0,05)$.

\section{RESULTADOS Y DISCUSIÓN}

\section{Población de plantas de frijol durante la etapa ve- getativa}

La población de plantas durante la etapa vegetativa en ambos sistemas de labranza, fue en promedio de 9,37 plantas/m lineal en labranza mínima, y de 9,55 plantas en labranza convencional (Figura 1). 


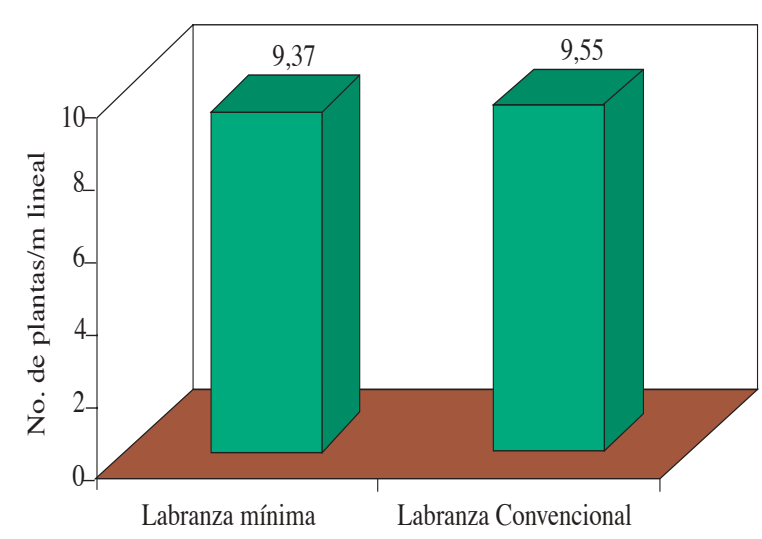

Figura 1. Población de plantas de frijol sembrado en labranza mínima y en labranza convencional obtenido durante la etapa vegetativa. Santa Cecilia, Los Chiles, Costa Rica. 2000.

De acuerdo a lo anterior, se obtuvo una densidad de 176.803 plantas/ha en labranza mínima, y de 180.213 plantas/ha en labranza convencional. Esta diferencia en densidad de plantas de frijol entre ambos sistemas de labranza no es relevante y no se atribuyó al sistema de labranza.

Por otra parte, la diferencia entre la cantidad de semilla sembrada y el número de plantas de frijol obtenido en ese estado fenológico (diferencia de un $25 \%$ aproximadamente), se atribuye a pérdidas en semilla que no germina y a posibles problemas fitosanitarios, principalmente patógenos e insectos de suelo que normalmente afectan el cultivo durante los primeros días de desarrollo.

\section{Población y peso seco de malezas}

El sistema de labranza es una de las prácticas que más afecta el manejo de malezas. La labranza convencional tiene la ventaja que se puede realizar control de malezas, principalmente malezas anuales, aunque las malezas perennes es factible controlarlas con la rastra en la época seca. Esto disminuye, aunque no elimina, la necesidad de aplicar otras técnicas de combate a través del ciclo de cultivo.

La labranza convencional elimina las malezas que han germinado, sin embargo, el mismo implemento de labranza lleva a la superficie del suelo, proveniente de capa inferiores, semillas de malezas que ya han superado el estado de latencia y germinan sin ningún problema al estar expuestas a condiciones favorables de humedad y temperatura.
En un sistema de labranza mínima, el rastrojo (material seco) de malezas y del cultivo anterior, no solo favorece una mayor retención de humedad y menor pérdida de agua por evaporación, sino que también como cobertura se convierte en una barrera física que limita en gran medida la germinación y crecimiento de las malezas (Figura 2). En esta figura se trata de rastrojo de gramíneas, principalmente Rottboellia cochinchinensis, que tienen un proceso de descomposición más lento por su contextura más fibrosa que otras familias de malezas.

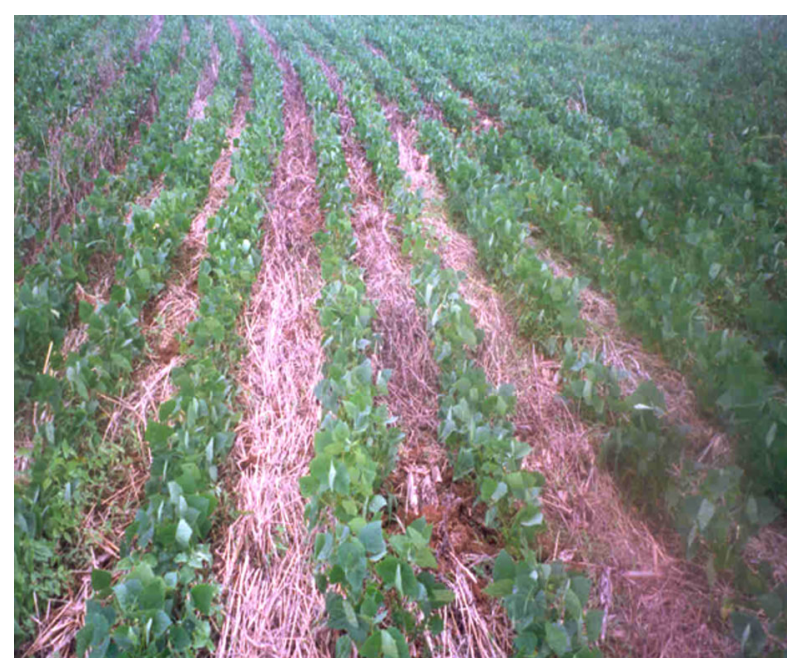

Figura 2. Rastrojo de malezas utilizado como cobertura en el cultivo de frijol. Santa Cecilia, Los Chiles, Costa Rica. 2000.

La Richardia scabra, Rottboellia cochinchinensis y Spermacoce spp fueron las malezas más dominantes en el frijol sembrado en ambos sistemas de labranza. Hubo presencia de arroz (Oryza sativa) que es arroz voluntario que germinó de la cosecha anterior, dado que en esta finca, al igual que otras de la zona, es común la rotación entre ambos cultivos. También se observó la presencia de Digitaria sp y Echinocloa colona, que son malezas comunes en el cultivo de arroz.

Se debe indicar que en la zona las malezas de mayor importancia en el cultivo de frijol son Rottboellia cochinchinensis, Baltimora recta y Melampodium sp, no obstante, estás últimas especies no estuvieron presentes en el área experimental o al menos no se presentaron en los sitios de muestreo al momento de la evaluación. Al final del ciclo de cultivo, sí se observaron estas malezas pero en una baja incidencia. Por otra parte, se obtuvo una mayor población de malezas en el sistema de labranza convencional (Figura 3), lo que demuestra el efecto de la mecanización en la incidencia de malezas anuales. 


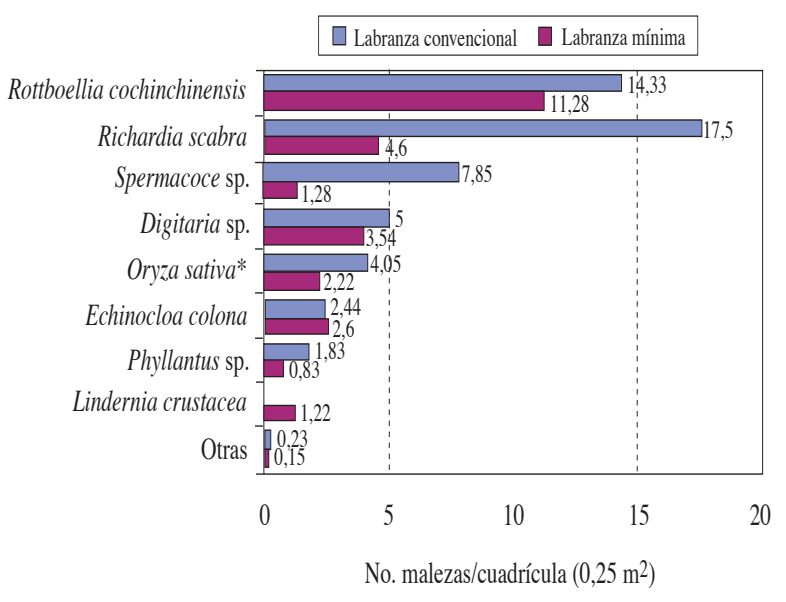

Figura 3. Población de malezas obtenida en el cultivo de frijol sembrado en labranza mínima y en labranza convencional. Santa Cecilia, Los Chiles. Costa Rica. 2000.

Esta finca tiene alrededor de 12 años de sembrarse frijol consecutivamente, no obstante, esta fue la primera vez que se sembró este lote en labranza mínima. Esta relación hace suponer que la población de malezas en una área con características similares debe ser homogénea, es decir, que tanto en el lote de labranza mínima como de labranza convencional podrían estar las mismas especies y en una población muy similar, sobre todo que el área en conjunto es relativamente pequeña. Esto sugiere que existe un efecto del sistema de labranza sobre la población de malezas, en donde el rastrojo de la cosecha anterior ejerció una barrera física que impidió o retrasó la germinación de semillas de malezas en el sistema de labranza mínima.

Por otra parte, inmediatamente después de la cosecha se determinó el peso seco de las malezas. El resultado obtenido fue congruente con el comportamiento de la población de malezas mencionado anteriormente. Se obtuvo un promedio de $37,6 \mathrm{~g}$ de malezas/cuadrícula de $0,25 \mathrm{~m}^{2}$ en labranza mínima, mientras que en labranza convencional se obtuvo un promedio de $46,4 \mathrm{~g}$ de malezas/cuadrícula (Figura 4). A pesar de la alta diferencia de campo en el peso seco de malezas, no fue significativa estadísticamente de acuerdo a la prueba de $t$ student $(\mathrm{P} \leq 0,05)$ debido probablemente a la alta variabilidad de los datos $(\mathrm{CV}=50,74 \%)$.

\section{Incidencia del gusano barrenador de la vaina Maru- ca testulalis}

En este ciclo de cultivo no hubo daño de ésta ni de otras plagas (Diabrotica spp, Empoasca kraemeri, Stig- mene acrea) debido a que el clima imperante de alta precipitación pluvial no fue una condición favorable para las plagas insectiles que normalmente afectan el frijol en esta región.

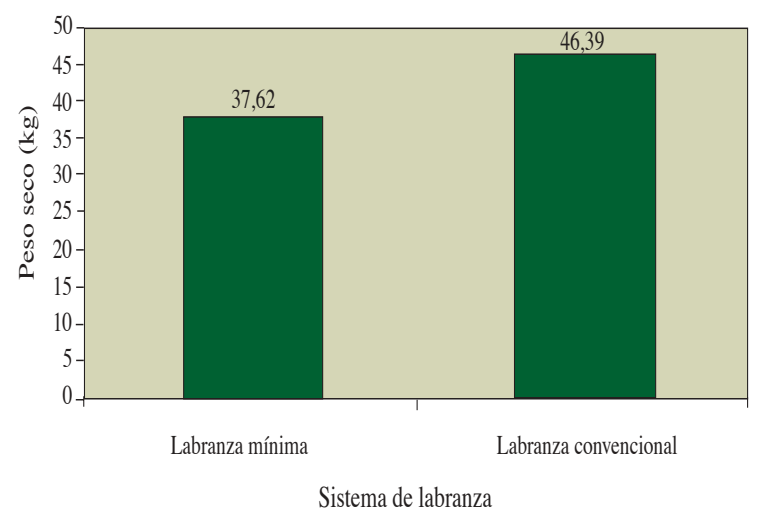

Figura 4. Peso seco de malezas obtenido en el cultivo de frijol sembrado en labranza mínima y en labranza convencional. Santa Cecilia, Los Chiles. Costa Rica. 2000.

\section{Incidencia y severidad de mustia hilachosa (Thana- tephorus cucumeris)}

La mustia hilachosa es considerada uno de los principales factores limitantes de la producción frijolera en las zonas húmedas y cálidas del trópico. En nuestro país es una enfermedad endémica que puede causar hasta el $90 \%$ de pérdidas si no se toman las medidas de control necesarias (CIAT 1985).

La incidencia de mustia en el sistema de labranza convencional fue de un 52,2\%, mientras que en labranza mínima fue de un 42,8\% (Figura 5), no obstante, esta diferencia no fue significativa estadísticamente en una probabilidad menor a $5 \%$, pero si fue significativa en una probabilidad menor al $10 \%$.

Por otra parte, según la escala de severidad del CIAT 1985, se obtuvo una mayor severidad en el sistema de labranza convencional $(1,22)$, mientras que en labranza mínima la severidad fue de 0,83 (Figura 6).

Esta diferencia fue significativa de acuerdo a la prueba de $t$ student, $\mathrm{P} \leq 0,05$.

En ambas situaciones podría ser que la menor incidencia y severidad obtenida en labranza mínima se deba al efecto de la cobertura al disminuir el salpique de la lluvia, que es una de las formas de diseminar la enfermedad. 


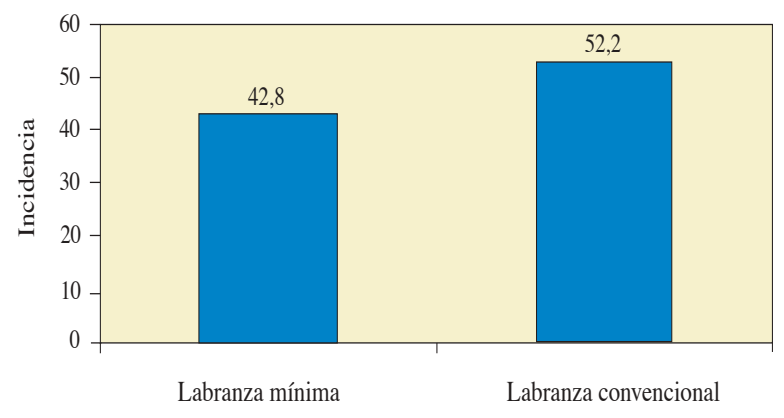

Sistema de labranza

Figura 5. Incidencia (\%) de mustia hilachosa obtenida en el cultivo de frijol sembrado en labranza mínima y en labranza convencional. Santa Cecilia, Los Chiles. Costa Rica. 2000.

\section{Número de vainas por planta y de granos por vaina}

En el sistema de labranza mínima se observaron plantas más vigorosas y de mayor desarrollo vegetativo. Lo anterior se reflejó en el número de vainas por planta, en donde, en labranza mínima se obtuvo un promedio de 7,25 vainas/planta, mientras que en labranza convencional se obtuvo 5,67 vainas/planta (Figura 7). Esta diferencia fue significativa de acuerdo a la prueba de $\mathrm{t}$ student, $\mathrm{P} \leq 0,05$.

Por otra parte, en el número de granos/vaina en el sistema de labranza mínima se obtuvo un promedio de 5,8 granos por vaina, mientras que en labranza convencional se obtuvo un promedio de 5,2 granos por vaina (Figura 8). Esta diferencia fue significativa de acuerdo a la prueba de $t$ student, $\mathrm{P} \leq 0,05$.

\section{Rendimiento}

Para obtener el rendimiento se cosecharon ambas parcelas en su totalidad. En el sistema de labranza

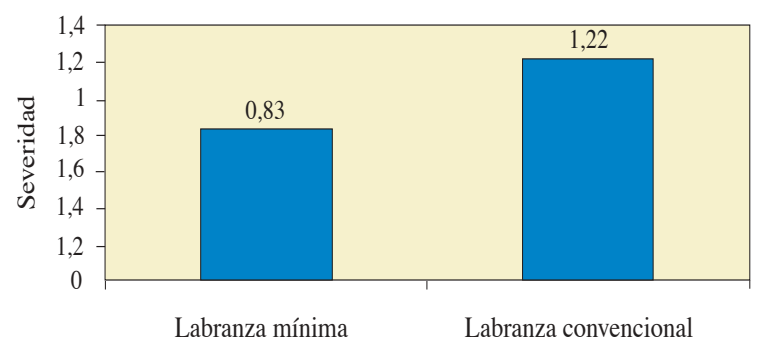

Sistema de labranza

Figura 6. Severidad de mustia hilachosa obtenida en el cultivo de frijol sembrado en labranza mínima y en labranza convencional. Santa Cecilia, Los Chiles. Costa Rica. 2000.

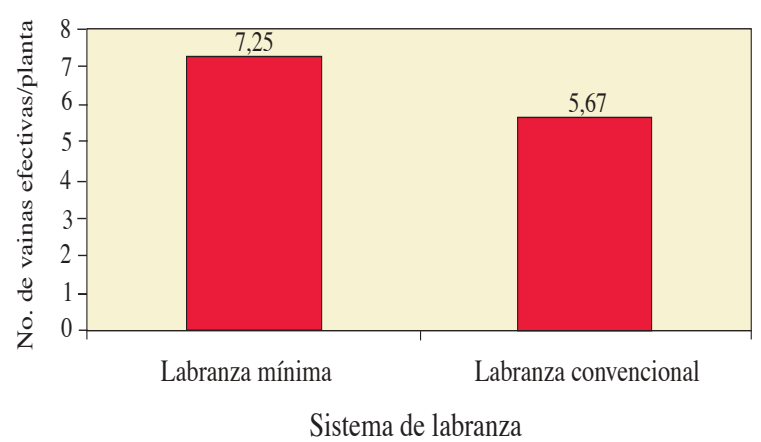

Figura 7. Número de vainas efectivas por planta obtenido en el cultivo de frijol sembrado en labranza mínima y en labranza convencional. Santa Cecilia, Los Chiles. Costa Rica. 2000.

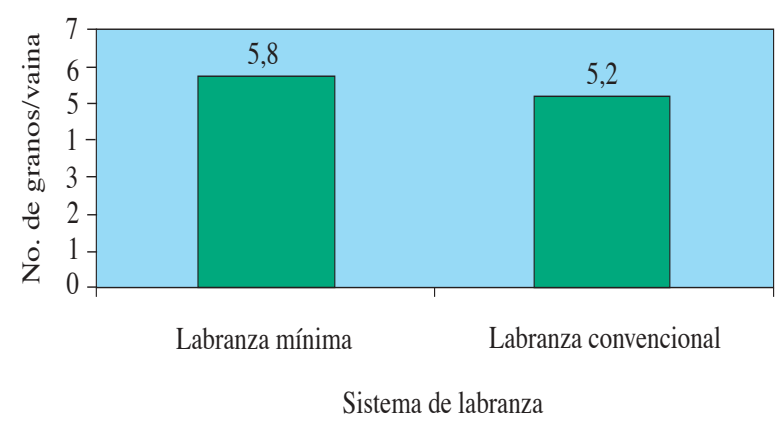

Figura 8. Número de granos por vaina obtenido en el cultivo de frijol sembrado en labranza mínima y en labranza convencional. Santa Cecilia, Los Chiles. Costa Rica. 2000.

mínima se obtuvo un rendimiento de $930 \mathrm{~kg} / \mathrm{ha}$, mientras que en labranza convencional el rendimiento fue de $676 \mathrm{~kg} / \mathrm{ha}$ (Figura 9). Es importante resaltar que esa diferencia en producción no fue afectada por la cantidad

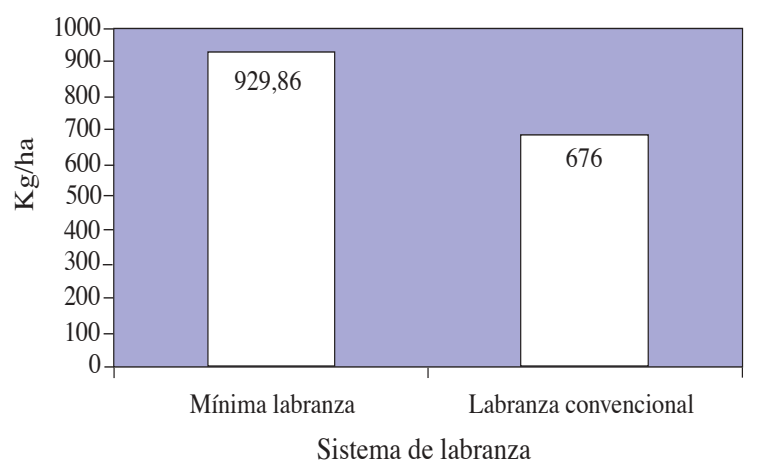

Figura 9. Rendimiento del cultivo de frijol sembrado en labranza mínima y en labranza convencional. Santa Cecilia, Los Chiles. Costa Rica. 2000. 
de plantas por metro o por área, puesto que en ambos sistemas de labranza el número de plantas fue similar (Figura 1). Por otra parte, este resultado fue congruente con el número de vainas efectivas por planta y número de granos por vaina, en donde en el sistema de labranza mínima se obtuvo una mejor respuesta.

\section{CONCLUSIONES}

La población de plantas de frijol evaluada durante la etapa vegetativa fue de 9,37 plantas/m lineal en labranza mínima y de 9,55 plantas/ $\mathrm{m}$ lineal en labranza convencional. Esta diferencia no fue significativa y no se atribuye al sistema de labranza.

Las especies Richardia scabra, Rottboellia cochinchinensis y Spermacoce sp fueron las malezas más dominantes en el cultivo en ambos sistemas de labranza; no obstante, se obtuvo una mayor población de malezas en el sistema de labranza convencional lo cual demuestra el efecto de la mecanización en la incidencia de malezas anuales.

En un sistema de labranza mínima la cobertura del rastrojo (material seco) de malezas y del cultivo anterior se constituyó en una barrera física que limitó directamente la germinación y crecimiento de las malezas.

El peso seco de malezas fue de $37,6 \mathrm{~g}$ de malezas/cuadrícula de $0,25 \mathrm{~m}^{2}$ en labranza mínima, y de $46,4 \mathrm{~g}$ en labranza convencional. Esta diferencia no fue significativa estadísticamente.

No hubo incidencia del gusano barrenador de la vaina (Maruca testulalis), debido a que el clima imperante de alta precipitación pluvial no fue una condición favorable para ésta y otras plagas insectiles que normalmente afectan el cultivo de frijol en la región.

La incidencia de mustia en labranza mínima fue de un $42,8 \%$ y en labranza convencional fue de $52,2 \%$; de igual forma hubo una mayor severidad de la enfermedad en el sistema de labranza convencional $(1,22)$, comparado con la severidad obtenida en labranza mínima $(0,83)$. Esta diferencia quizás se deba al efecto de la cobertura en labranza mínima al disminuir el salpique de la lluvia.

En labranza mínima se obtuvo un promedio de 7,25 vainas/planta y de 5,8 granos por vaina, mientras que en labranza convencional se obtuvo 5,67 vainas/planta y de 5,2 granos por vaina. En ambos casos la diferencia fue estadísticamente significativa.

En labranza mínima se obtuvo un rendimiento de $930 \mathrm{~kg} / \mathrm{ha}$, mientras que en labranza convencional el rendimiento fue de $676 \mathrm{~kg} / \mathrm{ha}$.

\section{LITERATURA CITADA}

ALTIERI, M. 1985. Bases científicas de la agricultura alternativa. Editorial Interamericana. Santiago, Chile. 173 p.

CENTRO DE INVESTIGACIÓN EN AGRICULTURA TROPICAL (CIAT). 1985. Frijol: investigación y producción. Compilado y editado por: Marceliano López, Fernando Fernández y Aart van Schoonhoven. Cali, Colombia, CIAT. 419p.

DERPSCH, R. 2000. Importancia de la siembra directa para obtener la sustentabilidad de la producción agrícola. En documento Proyecto MAG/FAO. Agricultura conservacionista: soluciones para continuar en el negocio.

GASSEN, D; GASSEN, F. 1996. Plantio direto o camino do futuro. Brasil. A Idea Sul Editora. 207 p.

HOLDRIDGE, L. 1987. Ecología basada en zonas de vida. San José, C.R., ICA. 216 p.

LEDESMA, E. 2002. Caracterización de la actividad frijolera con énfasis en la subregión de Santa Rosa de Pocosol. Cosecha 2000/2001. San Carlos, Costa Rica. C. N. p. $61 \mathrm{p}$.

PHILLIPS, S.; YOUNG, H. Agricultura sin laboreo: Labranza cero. Montevideo, UY, Hemisferio Sur. 223 p.

PITTY, A. 1997. Introducción a la biología, ecología y manejo de malezas. Tegucigalpa, HN. Zamorano. 300p.

UNGER, P.; LARYEA, K.; STEWART, B. 1995. Criterios para la selección de sistemas y prácticas de labranza. In Reunión Bienal de la Red Latinoamericana de Labranza Conservacionista (20, 1993, Guanare, Acarigua, Venezuela, VE, FONAIAP. p.118-146.

VEGA, J.; MUÑOZ, R.; PITTY, A. 1993. Evaluación de plagas, factores agronómicos y económicos de maíz y frijol en relevo bajo dos sistemas de labranza. Manejo integrado de plagas (Costa Rica) 26:13-20. 